\title{
A Walk-Through Programmed Robot for Welding in Shipyards
}

\author{
Marcelo H. Ang Jr.*, Wei Lin" and Ser-Yong Lim ${ }^{\#}$ \\ *Dept. of Mech. \& Prod. Eng. \\ \#Automation Technology Division, \\ National University of Singapore \\ 10 Kent Ridge Crescent; Singapore 119260 \\ mpeangh@nus.edu.sg \\ Gintic Institute of Manufacturing Technology \\ 71 Nanyang Drive; Singapore 638075 \\ wlin@gintic.gov.sg
}

\section{Corresponding Author}

Marcelo H. Ang Jr.

Dept of Mechanical and Production Engineering

National University of Singapore

Singapore 119260

mpeangh@nus.edu.sg

Autobiographical note of author:

Dr. Marcelo H. Ang, Jr. received the B.S. degrees (Cum Laude) in Mechanical Engineering and Industrial Management Engineering from the De La Salle University, Manila, Philippines, in 1981; the M.S. degree in Mechanical Engineering from the University of Hawaii at Manoa, Honolulu, Hawaii, in 1985; and the M.S. and Ph.D. degrees in Electrical Engineering from the University of Rochester, Rochester, New York, in 1986 and 1988, respectively. His work experience include heading the Technical Training Division of Intel's Assembly and Test Facility in the Philippines, research positions at the East West Center in Hawaii and at the Massachusetts Institute of Technology, and a faculty position as an Assistant Professor of Electrical Engineering at the University of Rochester, New York. In 1989, Dr. Ang joined the Department of Mechanical and Production Engineering of the National University of Singapore, where he is currently an Associate Professor. In addition to academic and research activities, he is actively involved in the Singapore Robotic Games as its founding chairman. His research interests span the areas of robotics, automation, computer control, and artificial intelligence applications.

Keywords:

- Walk-through programming

- Robotic welding

- Automation in shipyards

- Dynamic control 


\title{
A Walk-Through Programmed Robot for Welding in Shipyards
}

\author{
Marcelo H. Ang Jr.*, Wei Lin" and Ser-Yong Lim ${ }^{\#}$
}

\author{
*Dept. of Mech. \& Prod. Eng. \\ National University of Singapore \\ 10 Kent Ridge Crescent; Singapore 119260 \\ mpeangh@nus.edu.sg
}

\author{
\#Automation Technology Division, \\ Gintic Institute of Manufacturing Technology \\ 71 Nanyang Drive; Singapore 638075 \\ wlin@gintic.gov.sg
}

\begin{abstract}
:
Automating the welding process for the shipbuilding industry is very challenging and important, as this industry relies heavily on quality welds. Conventional robotic welding systems are seldom used because the welding tasks in shipyards are characterised by non-standardised workpieces which are large but small in batch sizes. Furthermore, geometries and locations of the workpieces are uncertain. To tackle the problem, a Ship Welding Robot System (SWERS) has been developed for the welding process. Main features of the SWERS include a special teaching procedure that allows the human user to teach the robot welding paths at a much easier and faster pace. In addition, operation of the system is made easier through custom designed man-machine interface. Through this interface, only a few buttons need to be pressed to command the robot into different modes. Optimised welding parameters can be selected from a large database though a Graphical User Interface system.
\end{abstract}

\section{INTRODUCTION}

In today's demanding and competitive ship building and repair industry, new technology is very much needed and automation plays a key role to improving the productivity and quality of shipyards. Welding is fundamental task in shipyards and marine/offshore companies. It is used in building and repairing structures. Fig 1 shows a typical panel consisting of a steel plate (base) and a number of vertically mounted stiffeners and webs. The webs are welded perpendicularly across the stiffeners, after the stiffeners are welded onto the panel. Welding of the stiffeners vertically onto 
the plates is a straightforward process using current automated welding systems. The stiffeners are long and straight, which makes automated welding easy. However, welding webs require much complicated welding motions to overcome access restrictions, as shown in Fig. 2. Each workpiece has a unique shape and dimension with some repeated patterns. There are variations in the size of the webs, stiffeners and plates. These practical aspects make the use of traditional robotic or automated welding systems difficult.

Robotic welding is very attractive because of its robustness and manipulability (Weston, 1989). It has been recognized as the next step in technological advancement of shipyards (Skjolstrup, 1994). There are many commercially available robotic welding systems and they have been applied to shipyards. One notable one is the robotic system of Odense Steel Shipyard Ltd in Denmark, wherein the robotic welding system is integrated into a CAD system and robots are programmed offline. Offline programming systems require the availability of CAD data describing the workpieces to be welded. A model of the robot and the welding process is then simulated in the computer together with a CAD model of the workpieces and environment. With a simulation environment, the robot program can be developed offline and tested before it is downloaded or implemented in the actual robot. Offline robot programming systems therefore require an accurate description of the workpieces and layout of the environment. Almost all of these welding systems are not attractive to the Singaporean shipyards. The are many important reasons for this. Robotic welding systems are very complicated to use, they require a robot programmer and/or application engineer which shipyards do not have. The workforce typically consists of welding operators and supervisors from the region whose training is up to the high school level at best. Also, CAD data of plates, webs, stiffeners are not available. Part geometries are only available in manual drawings and this makes off-line programming technique for robot teaching not applicable (Roedsted, 1987). Furthermore, the workplace is very unstructured and locations of work pieces are always uncertain. CAD-based robotic solutions such as those in (Kangsanant, 1995) are not suitable. Offline robot programming systems (Buchal, 1989) are therefore not feasible because of this uncertainty and the unstructured nature of the environment. Another problem is the workpieces are very large. The errors contributed by the stiffener positioning and the panel sagging can be up to $\pm 5 \mathrm{~mm}$ and must be accommodated. 


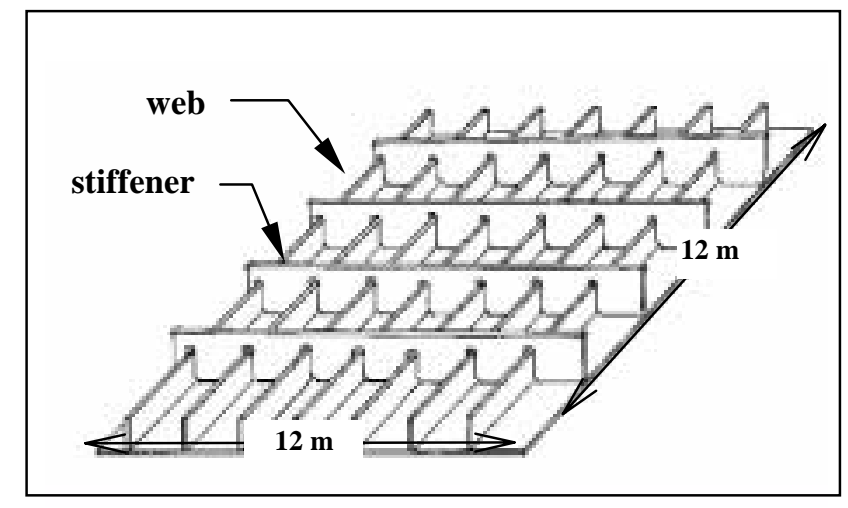

Fig. 1 Panel Workpiece

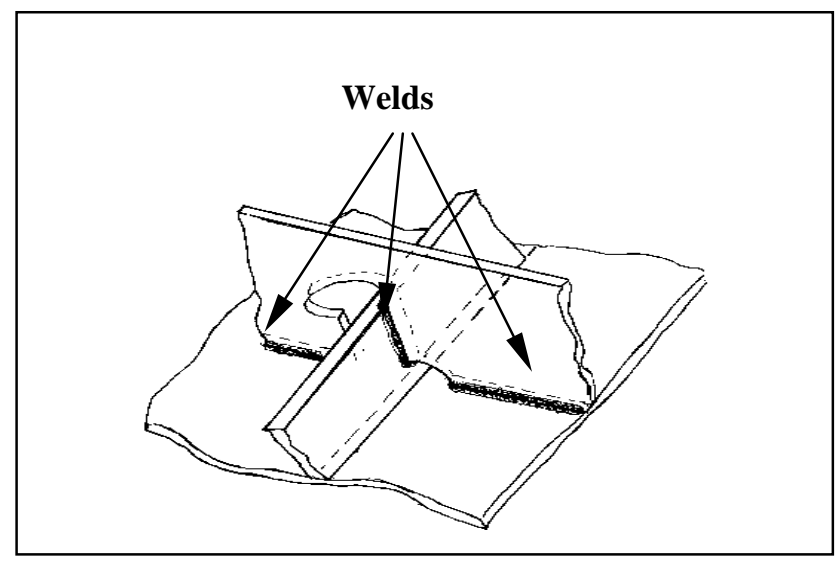

Fig. 2 Welding Lines

Web welding is therefore done manually and this process is very tedious. In addition to the slow manual welding, the manual welding process affects the quality of the weld because the welder has to stop and start the welding during the course of a welding path. Furthermore, the manual process results in unsteady welding motions with inconsistent welds along the seam. It is very common to have a lot of re-works due to bad quality welds. There are many other welding jobs in shipyards that are done manually because of similar reasons. In our work, we have developed a Ship Welding Robotic System (SWERS) that is easy to use and does not suffer from the aforementioned limitations. 
A very important requirement of our robotic solution is its ease of use, that is, it must be "idiot-proof" such that an unskilled or untrained worker can operate it out of his/her own "common sense". This is one of the most important design targets in our development of SWERS. Our approach is to develop a robotic system as a super tool for humans to use, rather than developing a completely autonomous system. The importance of human operators in man/machine teams has been recently recognized to be more useful that purely autonomous systems, especially so in welding (ABB, 1993).

In this paper, we present details of our SWERS. In Section 2, we describe the walk-through programming approach and the algorithms to enable walk-through motion capabilities in industrial robots. The walk-through algorithm is crucial to the ability to program the robot without the need for learning complicated robot commands and program syntax. In Section 3 we discuss the SWERS. Welding tests are discussed in Section 4 together with practical issues during production runs at Keppel Far East Levingston Shipbuilding Ltd. Section 5 presents the benefits and conclusions.

\section{WALK-THROUGH TEACHING METHOD}

The main feature SWERS is the walk-through (or direct teaching) method for robot programming. Here, the welder teaches the robot by guiding it once through the required welding motions at a much easier and faster pace, and as though he/she is doing the welding her/himself. The motion is recorded and the robot thereafter does the unpleasant job of welding by "playing back" the recorded motions. Continuous recording of the welding positions and motions, or user-initiated recording of critical positions and configurations are possible.

Walk-through teaching has been around for many years now and has also been referred to as "lead-through programming" (Groover, 1986). Groover classifies this into two kinds: powered lead-through and manual lead-through. Powered leadthrough is what we commonly see in today's industrial robots, where a teach-pendant is used to move joints of the robot such that the desired end-position and orientation in space is reached. In this mode of teaching, the motors of the robot are powered. In manual lead-through, the joint motors of the robot are not powered and left free with brakes turned off. The human operator can then physically move the robot links. Manual lead-through is therefore not feasible for robots with high gear ratios or robots whose configurations do not allow the joints to be released and powered down. Robots, whose joints move the links against gravity require constant power to maintain position, otherwise the links would drop. High gear ratios in the joints make the robot links hard to move physically, i.e., they are not backdrivable. Our approach is different in that 
the robot's motors are always powered to maintain the current joint positions and at the same time allowing the human operator to physically move the links with ease according to the operator's preferences. Thus our approach of walkthrough programming is applicable to any robot regardless of high gear ratios or joints operating against gravity. To the best of our knowledge, there is no industrial robot that can achieve this.

A 6-axis force-torque sensor is mounted on the welding torch through a custom-built walk-through teaching (WTT) handle. The operator grasps the WTT handle (Fig. 3) and moves the welding torch naturally to position it in the required welding positions. The sensor senses the force and moments exerted by the operator's hand. The controller then commands the robot to move in response to the sensed forces. To achieve this, it is important to be able to control the dynamic behavior of the robotic manipulator, or to control the "impedance" of the manipulators. Impedance control was first introduced by Hogan (Hogan, 1985) as a natural way of dealing with contact forces. Let $\mathbf{f} \varepsilon \Re^{6}$ be the forces and moments acting on the manipulator by the environment (e.g., operator's hand). Then the desired motion response $\mathbf{x}_{\mathbf{a}} \varepsilon \mathfrak{R}^{6}$ can be specified according to

$\mathbf{f}=\mathbf{J} \ddot{\mathbf{x}}_{a}+\mathbf{B} \dot{\mathbf{x}}_{a}+\mathbf{K} \mathbf{x}_{a}$

where $\mathbf{J}, \mathbf{B}$ and $\mathbf{K}$ (all $\varepsilon \Re^{6 \times 6}$ ) are the desired mass, damping, and stiffness of the manipulator as seen from the operational space of the robot (Khatib, 1987), where the forces act on the robot. The motion $\mathbf{x}_{\mathbf{a}}$ can be viewed as a motion adjustment from the current robot position. The control objective is to regulate the time evolution of $\mathbf{x}_{\mathbf{a}}$ according to the desired dynamic behavior specified in Eq. (1). The dynamic properties can be specified according to the desired task to be achieved (Ang, 1995). For example, the manipulator can be made to behave with an apparent mass $\mathbf{J}$ that is very different from the true mass properties of the arm, thus influencing the response of the manipulator to external forces. Or $\mathbf{B}$ can be specified to simulate manipulator behavior underwater, $\mathbf{K}$ can be specified to make the manipulator behave like a spring with different stiffnesses. Theoretically, all the dynamic parameters $\mathbf{J}, \mathbf{B}$ and $\mathbf{K}$ can be adjusted to values different from the physical parameters of the manipulators by just regulating the motion response $\mathbf{x}_{\mathbf{a}}$ to sensed forces $\mathbf{f}$. Compliant motion control is a special case where the control objective focuses upon the control of the stiffness K (Salisbury, 1980). There are however, many practical problems. These include actuator limits, noisy sensor readings, stability problems, and the performance of the control algorithms is limited by the ability to achieve the desired response $\mathbf{x}_{\mathbf{a}}$. 
The control implementation to achieve the desired response in Eq (1) can be done in a number of ways. The model-based approach computes the joint torques that make the robot follow the impedance model using position, velocity and possibly acceleration feedback. In such implementations, it is necessary to compensate explicitly for the robot dynamics. In general, it is difficult to obtain a good robot and actuator model. The resulting controller can be rather complicated and difficult to implement in real-time. For practical implementation, the non-model-based approach provides a simple alternative and is very attractive for implementation in industrial robots. The resulting controllers are relatively simpler since they do not include a dynamic model for the robot. There are several ways of implementing this idea however, and the performance of these controllers is always dependent on the quality of the underlying position control system (Tan, 1987).

The straightforward implementation of impedance control, proposed by Lawrence (Lawrence, 1988) and Pelletier and Doyon (Pelletier, 1994), uses force feedback only to modify the input position. It assumes that the position tracking is perfect and consequently does not try to correct any errors by using state feedback from the system. In addition, contact stability requires significantly overdamped impedance behaviour for stable contact with a stiff environment and thus it is unable to provide soft impedances, that is, small stiffness and damping.

An alternative approach to the straightforward method is the velocity method, proposed by Pelletier and Doyon (Pelletier, 1994). In this approach, position and acceleration feedback are utilised in addition to force feedback. Using the velocity method ensures that the steady state tracking error will be zero. This method can remain stable at high environment stiffness by using high values of damping (Tan, 1987).

For SWERS, we are interested in providing the robot with the walk-through programming capability. We want the operator to be able to move the arm through the WTT Handle on the sensor (see Fig. 3) as if he is operating in a zero-gravity environment. This means the robot behaves like it's floating in space and can be easily moved. To achieve this we specify a zero stiffness $(\mathbf{K}=\mathbf{0})$ and the values of $\mathbf{J}$ and $\mathbf{B}$ can be specified according to the operator's preferences. If the operator feels the robot is too heavy, a lower value of $\mathbf{J}$ can be specified. If the robot feels too fast, a high damping is specified. For accurate positioning of the robot, a relatively high value of $\mathbf{B}$ may be desired. 


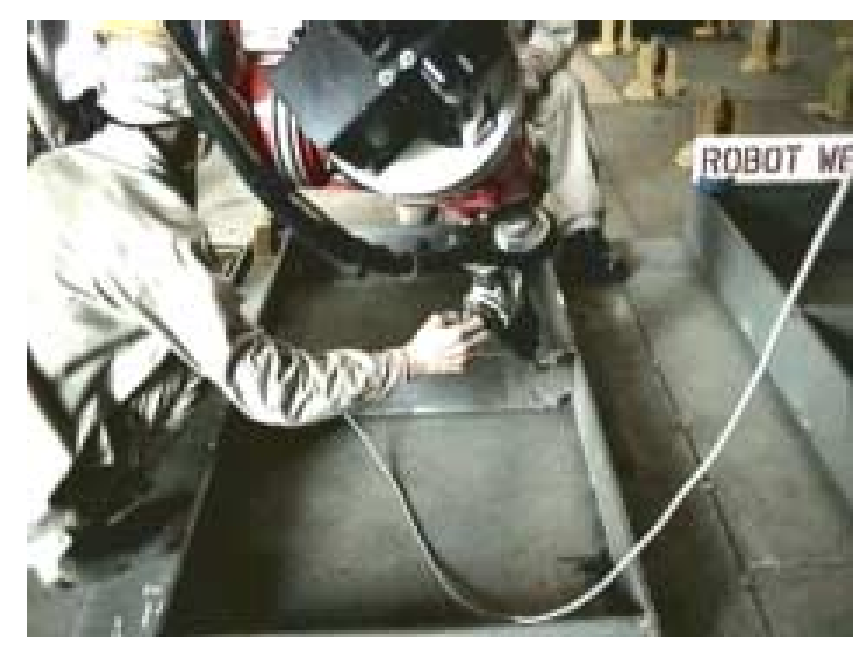

Fig. 3 Robot Teaching

To realize the control using an industrial robot, such as the REIS RV6 six dof robot, which is used for SWERS, we employ the non-model based control. With non-model based control, we do not need to identify the dynamic model of the robot, and we can use the stable position control performance provided by the robot itself. In this way, however, we are limited by the performance of the robot controller. But for our objective of walk-through programming, this would suffice because we need not accurately regulate the motion response of the robot during walk-through teaching.

The adjustment trajectory is continuously computed in the high level servo loop by sensing the forces and solving Eq. (1). Note that Eq. (1) is a linear differential equation with an analytical solution. In discrete time implementation, the relationship can be approximated in the form

$$
\mathbf{J} \frac{\left(\mathbf{x}_{a}\right)_{i}-2\left(\mathbf{x}_{a}\right)_{i-1}+\left(\mathbf{x}_{a}\right)_{i-2}}{d t^{2}}+\mathbf{B} \frac{\left(\mathbf{x}_{a}\right)_{i}-\left(\mathbf{x}_{a}\right)_{i-1}}{d t}+\mathbf{K}\left(\mathbf{x}_{a}\right)_{i}=\mathbf{f}
$$

where $d t$ is the sample time for the higher level force servo loop. This gives

$$
\left(\mathbf{x}_{a}\right)_{i}=\left(\frac{\mathbf{J}}{d t^{2}}+\frac{\mathbf{B}}{d t}+\mathbf{K}\right)^{-1}\left(\mathbf{f}+\mathbf{J} \frac{2\left(\mathbf{x}_{a}\right)_{i-1}-\left(\mathbf{x}_{a}\right)_{i-2}}{d t^{2}}+\mathbf{B} \frac{\left(\mathbf{x}_{a}\right)_{i-1}}{d t}\right)
$$

Generally, $\mathbf{x}_{\mathbf{a}}$ is expressed in base frame $\{B\}$ and is represented by differential displacements in position $\left(d_{x}, d_{y}, d_{z}\right)$ and orientation $\left(\delta_{x}, \delta_{y}, \delta_{z}\right)$ expressed in base coordinates: 
$\mathbf{x}_{a}=\left[\begin{array}{llllll}d_{x} & d_{y} & d_{z} & \delta_{x} & \delta_{y} & \delta_{z}\end{array}\right]^{\mathrm{T}}$

Therefore, the force must also be represented in $\{B\}$. To do so the force must be mapped to $\{B\}$ from the WTT- frame $\{T\}$, which is mapped from the sensor frame $\{S\}$ (where the interaction force is measured).

The new commanded position $\mathbf{x}_{\mathbf{c}}$ is then computed by adding the required adjustment motion $\mathbf{x}_{\mathbf{a}}$ to the current position and orientation $\mathbf{x}$ of the robot:

$\mathbf{x}_{c}=x+x_{a}$

Eq. (5) is in terms of operational coordinates $\left(\varepsilon \mathfrak{R}^{6}\right)$. In terms of the homogenous transformation matrices, Eq. (5) becomes (Paul, 1981):

$$
\begin{aligned}
{ }^{\boldsymbol{B}} \mathbf{T}_{\boldsymbol{c}} & ={ }^{\boldsymbol{B}} \mathbf{T}_{\boldsymbol{E}}+\Delta^{\boldsymbol{B}} \mathbf{T}_{\boldsymbol{E}} \\
& =\left[\begin{array}{llll}
1 & 0 & 0 & \boldsymbol{d}_{\boldsymbol{x}} \\
0 & 1 & 0 & \boldsymbol{d}_{\boldsymbol{y}} \\
0 & 0 & 1 & \boldsymbol{d}_{z} \\
0 & 0 & 0 & 1
\end{array}\right]\left[\begin{array}{cccc}
1 & -\delta_{z} & \delta_{\boldsymbol{y}} & 0 \\
\delta_{z} & 1 & -\delta_{x} & 0 \\
-\delta_{\boldsymbol{y}} & \delta_{x} & 1 & 0 \\
0 & 0 & 0 & 1
\end{array}\right]{ }^{\boldsymbol{B}} \mathbf{T}_{E}
\end{aligned}
$$

where ${ }^{\mathrm{B}} \mathrm{T}_{\mathrm{c}},{ }^{\mathrm{B}} \mathrm{T}_{\mathrm{E}}$ and $\Delta$ are the homogenous transformation forms of $\mathbf{x}_{\mathbf{c}}, \mathbf{x}_{\mathbf{E}}$ and $\mathbf{x}_{\mathbf{a}}$ respectively. The robot is then commanded to move to this new position and orientation at each force servo tick. In our implementation, the force servo rate is $50 \mathrm{~Hz}$ (which corresponds to the reference trajectory update rate), while the low level motion servo rate of the robot controller is $500 \mathrm{~Hz}$. In can be noted that Eq. (1) is a linear differential equation whose solution can be analytically derived in closed form. Hence another approach is to explicitly solve for the time evolution of $\mathbf{x}_{\mathbf{a}}$ with the sense initial conditions at each servo tick. Our experience indicates no difference between the two approaches.

Besides the new robot programming method, a new man-machine interface (MMI) that includes custom designed teach pendants and Graphical User Interface (GUI) are developed. The MMI allows the welder to input the mode of motion and alter the welding parameters. 
The welding path tracking is further improved (from the taught path by the human user) through advanced sensing so as to obtain better quality welds.

\section{SYSTEM DESCRIPTION}

A picture of our SWERS is shown in Fig. 4. The main structure is a 3-axes gantry on which a robot (REIS RV6) is mounted upside down. The workspace of the robot is about 3 meters in diameter and the gantry moves the base of the robot in a work volume of $12 \mathrm{~m} \times 12 \mathrm{~m} \times 2 \mathrm{~m}$. The gantry was designed to accommodate the $12 \mathrm{~m} \times 12 \mathrm{~m}$ panel size requirements in Keppel FELS, a shipyard in Singapore. The whole gantry moves on a pair of tracks with length 16 m.

The long travel distances of the gantry enables the robot to cover a typical panel size of $12 \mathrm{~m}$ by $12 \mathrm{~m}$. Between the two tracks are an array of roller wheels mounted on the floor for facilitating the positioning of the panel plate for the welding operation.

Mounted together with the robot are the welding accessories such as the wire reel drum, the wire feeder and a torch cleaning station. The welding torch and a WTT Handle are equipped on the welding torch handle. The welding power source is placed on top of the gantry. The robot controller, Supervisory Console and the gantry controllers are housed in the air-conditioned Control Room at one end of the gantry. The Control Room moves with the gantry.

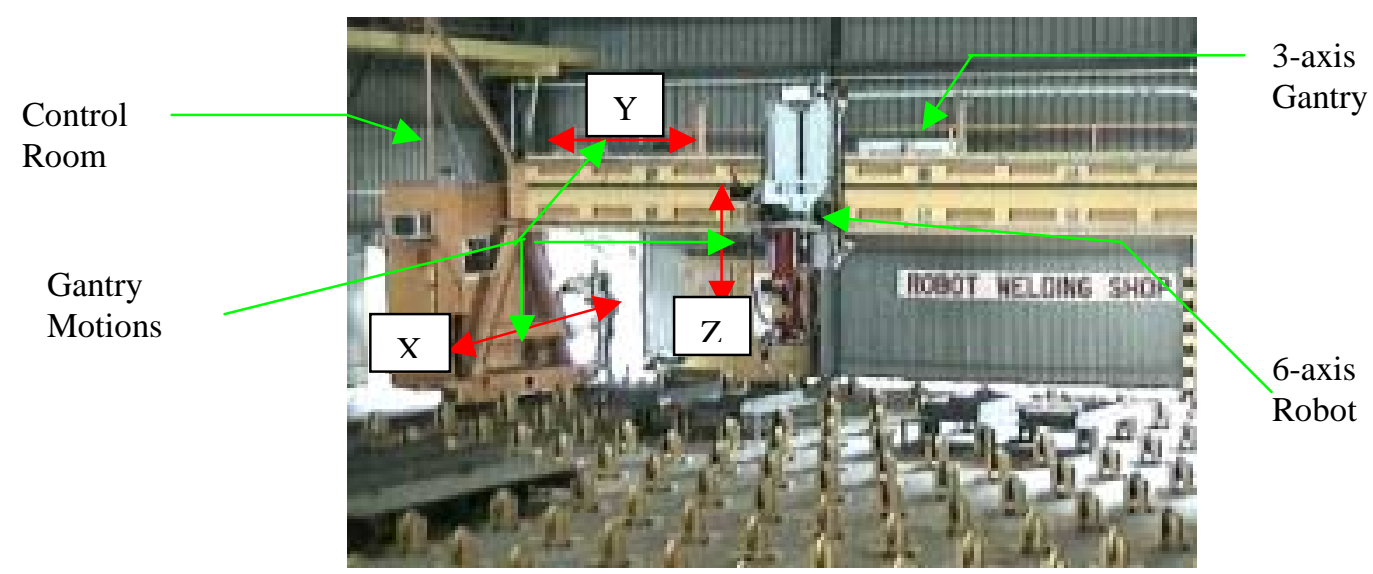

Fig. 4 The SWERS 
The SWERS is divided into four subsystems:

- Robot subsystem

- Welding Subsystem

- Man-Machine Interface Subsystem

- Gantry Subsystem

\section{Robot Subsystem}

A 6 degrees-of-freedom articulated robot arm as shown in Fig. 5 is used for the dextrous motion requirements. It covers a work envelope of $3 \mathrm{~m}$ diameter within which a WTT session can be carried out. A WTT session consists of a number of mini sessions each of them can be in the mode of "Teach-Weld-Teach-Weld" (TWTW) or "Teach-Weld-Weld" (TWW). The TWTW mode is employed for welding motions, which are not repeated through different sections of the panel. Hence each section has to be taught separately. The sequence of motions is then teach then weld for a section within the reach of the robot, followed by teach then weld on a different section, and so on. The TWW mode is for sections whose welding paths are the same as previous sections. Here, teaching of the complete welding path is only done once, and welding is done at different sections without the need to teach the welding path again. Only reference positions are taught for each new repeated section. The repeatability of the robot is $\pm 0.05 \mathrm{~mm}$ which is far better than the required welding accuracy.

Besides the robot arm, the robot subsystem also includes force/torque sensor, a Robot Teach Pendant and a robot controller. The force/torque sensor controller is VME based and is fully integrated in the robot controller. The Robot Teach Pendant is not normally used for the welding operation. It is replaced by two custom designed pendants for the new man-machine interface of the SWERS.

The main feature of the robot is the VME-based open architecture controller. The existing boards on the robot controller include a master CPU board, which is for the user programs and Robot Teach Pendant; slave CPU, for robot/motion control and welding control, a system I/O board; arc sensor interface board for weld seam tracking; and three servo boards, for the 6 joints of the robot arm. 


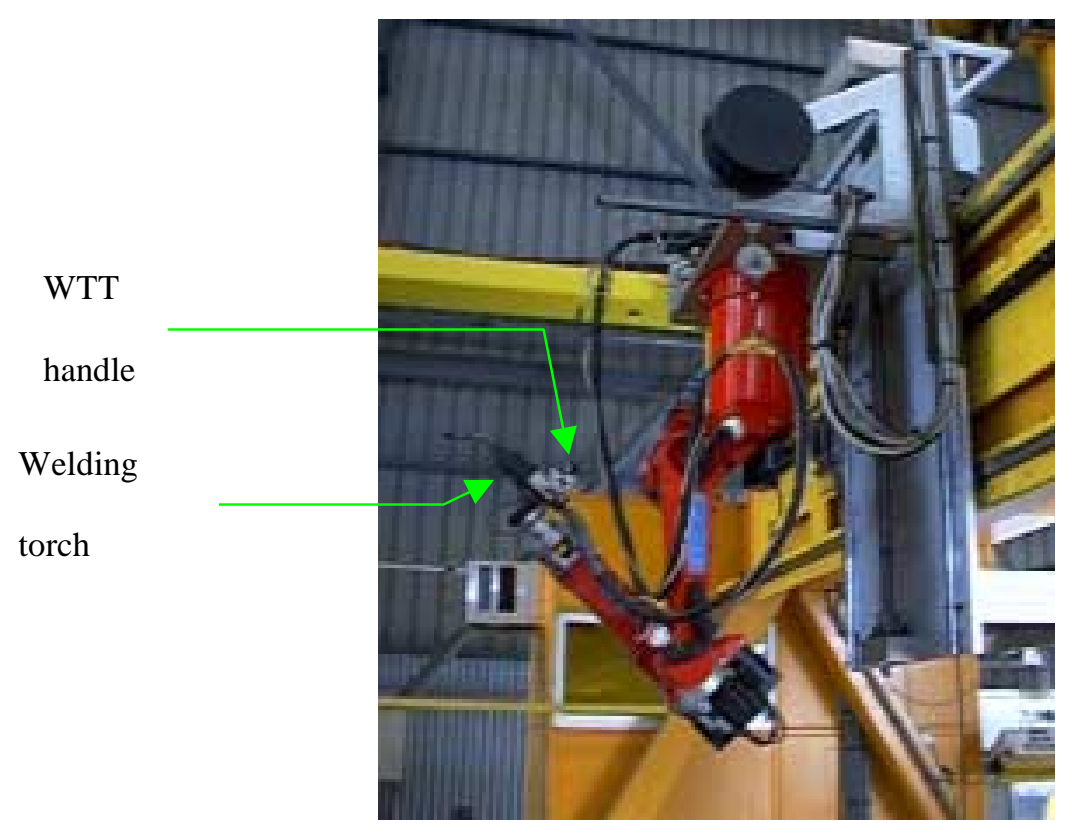

Fig. 5: REIS RV6 robot with our WTT handle

Another feature of the robot controller is that its co-ordinate interface protocol (from REIS robotics) is available. This allows lower level access to the robot control so that the WTT algorithm and MMI can be implemented.

A VME bus-based SPARC CPU board is interfaced with the robot controller as shown in Fig. 6. The SPARC CPU board is the platform for the development of the WTT and MMI. This board is configured as a slave and is plugged onto the VME bus of the robot controller. he communication between the robot controller and the SPARC controller is through the shared memory as there is no direct access to the teach pendant from the SPARC controller. This shared memory allows the total control of the robot arm as well as the welding system from the SPARC controller. 


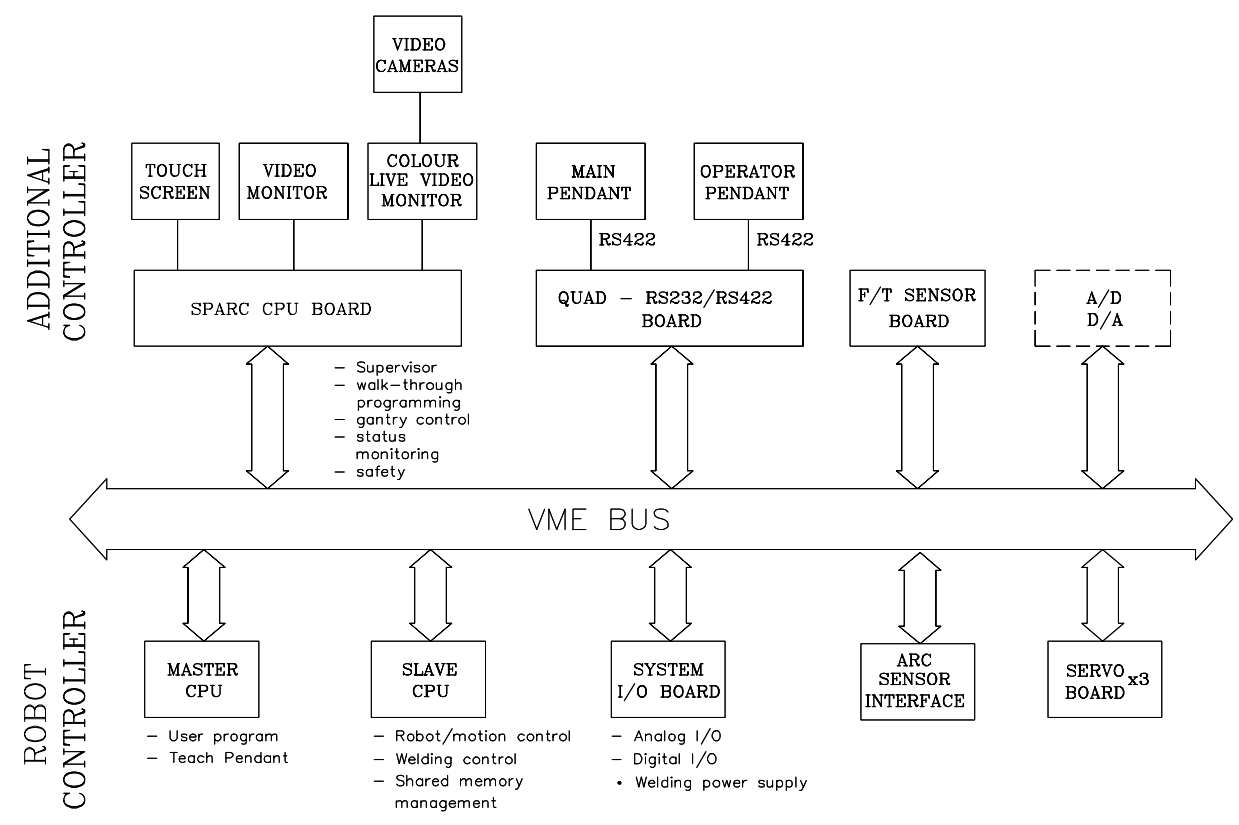

Fig. 6 Controller Hardware Architecture

\section{Welding Subsystem}

The welding system consists of a power source, a wire feed unit, a welding torch and a torch nozzle cleaning station. It also comes with a built-in seam tracking arc sensor for detection of welding path. It compensates errors arisen from workpiece distortion and tolerance.

The direct current inverter power source renders possible welding processes including the flux-cored wire arc welding used for the panel line welding. The power source is controlled by digital and analogue signals via the system I/O board in the robot controller.

By means of the push-pull control board, the motor in the torch housing is synchronously controlled to the drive motor of the wire feeding unit so that a constant arc will be produced during welding process. The welding torch is water cooled and comes with a escape mechanism to protect from any damages due to collision. 
A welding parameters database are created for the various welding conditions such as the application of arc sensor and various welding modes for the panel structures. This database contains optimised parameters obtained by studies and extensive experimentation. These include experimental studies of the coupling between weld process and arc sensor parameters. The welded samples have been through macro-etch inspection and certified for the welding quality. The welding parameters installed in the database for a particular type of wire and welding position can be easily selected from the database through a GUI. Depending on the taught motion during WTT, the optimum parameters are automatically selected from the database. For example, if the welding motion is vertical, the welding speed, arc sensor and other parameters for vertical welding are automatically selected. These parameters, however, can be overridden even during actual welding by the operator. Welding need not be stopped and parameter overrides can be effected on line during welding.

The welding parameters include the Welding Mode (Position), Leg Length, Wire Type, No. Of Passes, Welding Current, Welding Voltage, Welding Speed, Stick out Length, Oscillation Frequency, Oscillation Amplitude, and Oscillation Hold Time

\section{Gantry Subsystem}

The massive gantry has 3 axes of movements. It is driven by a pair of AC induction motors along the flow of the panel (X drive). The robot is transferred across the panel by an AC servomotor (Y drive). Due to the height of the webs and limited size of the robot work envelope, the robot has to be raised up ( $\mathrm{Z}$ drive) for the next welding zone. All motors are equipped with brakes and incremental encoders.

The communication between the robot controller and the gantry controller is through an RS232 interface. The gantry is able to index the robot at a number of points across and along the workpiece. The locations of these points are determined such that they ensure that the robot covers the entire required welding area. In order to ensure flexibility, the gantry can also move and stop anywhere the operator desires. In addition, the indexing positions of the gantry can be adjusted easily at the Main Pendant. Position errors of the gantry are compensated by the fine positioning of the robot arm during WTT. 


\section{Man-Machine Interface}

To facilitate the Walk-Through Teaching and to make the system easier to operate, custom-designed MMI software and hardware are developed.

The hardware of the MMI consists of a Main Pendant (MP), a User Pendant (OP) and a Supervisory Console (SC). As shown in Fig. 7, the OP is attached on the robot near the welding torch. It is a control pendant for WTT only. The MP and the SC are located outside the gantry. They are for overall control of the SWERS and data/parameter entry.

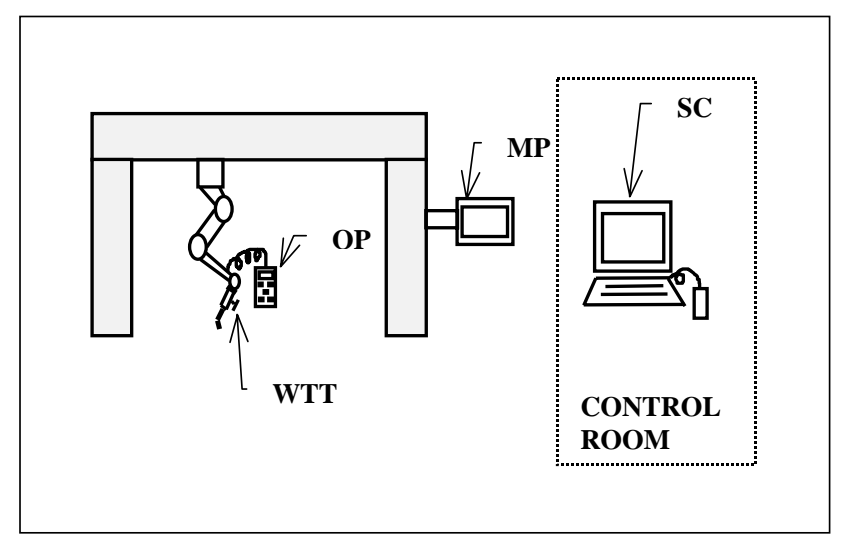

Fig. 7 MMI Hardware

The handle located at the welding torch is for WTT. The handle is designed under the considerations of ease in teaching and no interference during welding.

\section{Supervisory Console (SC)}

The Supervisory Console is located inside the Control Room of the SWERS. It consists of a monitor, a keyboard and a mouse. Upon start off the SWERS, the user firstly uses the SC to select and adjust the welding parameters according to the welding wire and/or leg length he desired to work on. A Graphical User Interface (GUI) is developed to facilitate the 
selection of the welding parameters. Figure 6 illustrates an example of the menus and dialog boxes of the GUI. It consists of a Main Menu for data file management, a Welding Parameters Editing Menu for parameter adjustments and a Status Window for data monitoring.

The GUI allows the user simply to click the mouse button to select the desired welding data of leg length, wire type, welding mode and number of passes. The selected data is matched with the default and optimised values of the welding parameters such as current, voltage, speed, oscillation etc. The user may adjust the values of the parameters if he/she so desired. The default values of the parameters are based on a welding study and are consistent with the welding specifications. Table 1 shows the fields in the welding database. The user can, however, add, delete, and change the records in the database. This ensures that the database grows and improves with experience. The user updates the data using the mouse and the keyboard.

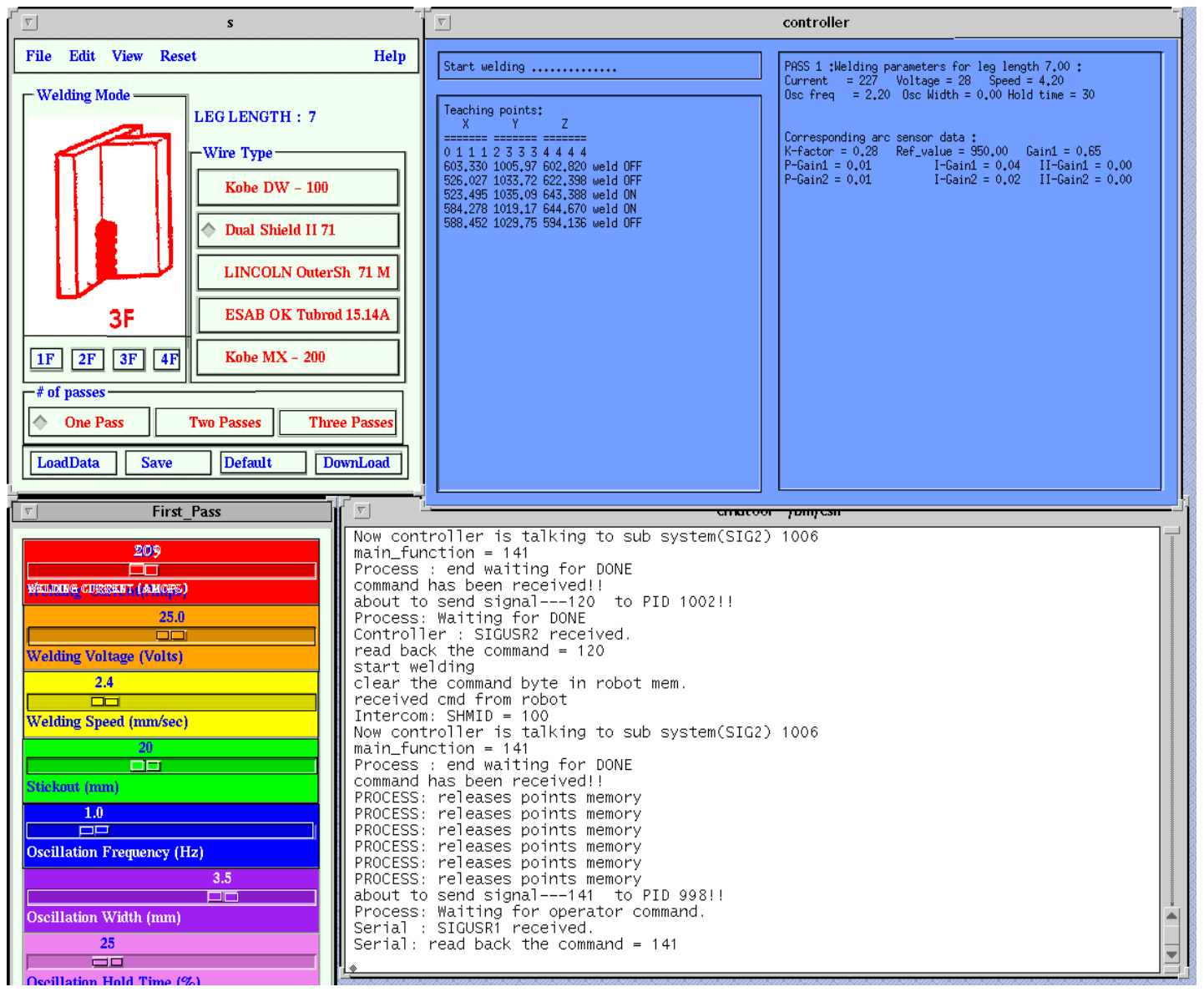

Fig. 8 GUI for Welding Parameters 


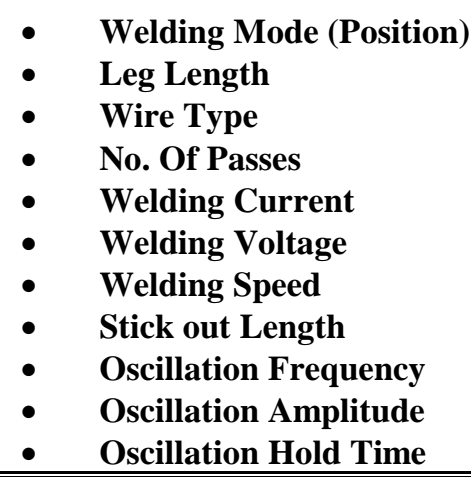

Table 1: Important Welding Process Parameters

The operator can see the actual welding using a video monitor that takes input from a remote camera mounted on the manipulator arm. Welding parameters can be changed on-line during actual welding without the need to stop the current welding operation. The GUI is developed using the Motif tool kit in the X-windows environment. It runs on the UNIX platform (Solaris 2.3), which is the operating system of the VME bus based controller.

\section{Main Pendant (MP)}

The MP located near the Control Room is a device for overall control of the whole welding system. It is the only device that commands and controls movements of the gantry. Using the MP, the operator can home the gantry, index the gantry, move the gantry, calibrate the robot, clean the welding torch, and select the desired teach modes and welding modes.

For safety purpose, it can only be operated when the OP is hanged up on the robot arm and no operator is in the welding area.

The MP consists of a touch screen terminal. The touch screen has the advantages of ease of use and programming flexibility. The screen displays the desirable buttons for the user to press at different states. It also displays the robot and welding status.

\section{User Pendant $(O P)$}

The OP is a hand-held device for the user to perform Walk-Through Teaching. (Fig. 9). It is activated by the MP located remotely from the robot. The OP is connected on the robot at a position near the welding torch. To begin the teaching, the 
user firstly removes the OP from the robot arm and a sensor detects that the OP is detached. After the teaching procedure, he/she attaches the OP back to the robot and all the buttons are then disabled. The user then leaves the welding area for the MP. This feature ensures that no user is around the robot when it is moved by the MP.

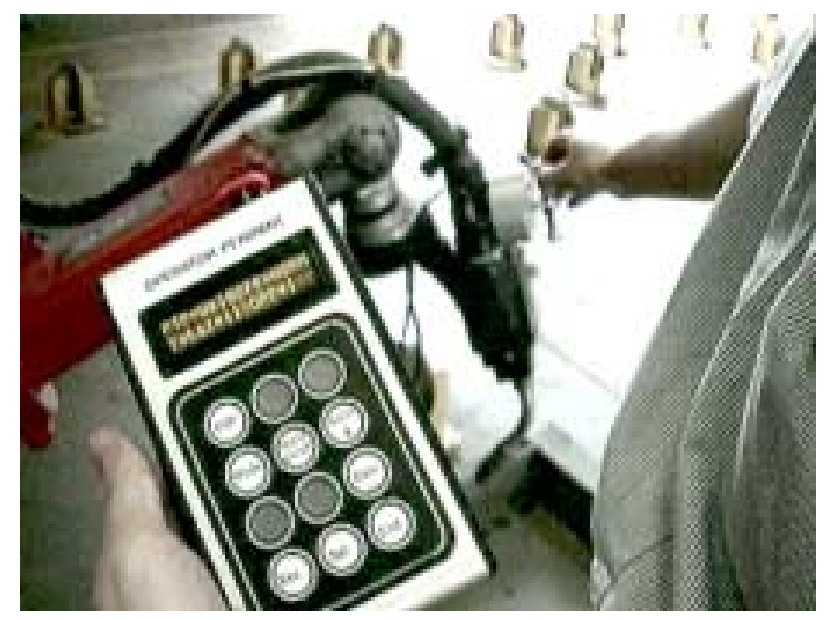

Fig. 9 Operator Pendant

As shown in Fig. 9, there are eight buttons and a 2x16 LCD on the OP. The LCD displays the current teaching state. The PTP button allows Walk-Through Teaching for Point-to-Point mode. As the operator leads the torch to the point to be recorded, he/she can select the mode of robot movement by pressing the Mode button. Three modes can be select with the toggle button, namely the Normal, Rotate and Translation modes. The Normal Mode allows the operator to rotate and shift the position of the torch. On the other hand, the Rotate Mode and the Translate Mode decouple the two modes allowing a easier movement either by rotating the torch about the Tool Center Point or just moving the torch position. The Gain + and Gain - buttons increases or decreases the gain of the robot speed. The operator can adjust the gain value for the easiness of moving the robot.

There are two states, namely Weld On and Weld Off. The user can teach the robot for the desired state by pressing the corresponding buttons as he/she traces the required robot path. The point just recorded can be deleted if he/she so desired. An additional option for continuous recording at user specified rates is possible, but not implemented in this first version of SWERS. 


\section{Safety Subsystem}

Generally, the overall system is designed and constructed under standard safety guidelines of industrial robots (PSB, 1990). Some of the safety features are as follows:

- Emergency Stops are available on the Reis Robot Controller, MAIN PENDANT and OPERATOR PENDANT.

- Software module of each subsystem includes exception handling routines which ensure that the software will not be aborted abnormally leaving the system in an unknown state.

- In "TEACH mode", the velocity of the robot is restricted at a safe speed and the robot cannot be moved on the gantry.

- The MAIN PENDANT is operational only when the TEACHING PENDANT and all other control stations are deactivated.

- A sensor is implemented to detect whether the OPERATOR PENDANT is detached from the robot.

- The program ensures that the robot is in up position when the robot moves in $\mathrm{X}$ or $\mathrm{Y}$ direction.

- Light curtain is installed for vertical safe-guarding of the robot work envelope.

\section{PERFORMANCE EVALUATION}

The SWERS have been certified and performance has been good. Certification includes extensive laboratory tests of welded specimens. These include hardness tests and micro-edge inspections, and macro-etch tests for cracks and porosity. Extensive experimental runs and demonstrations have been conducted. Typical runs involving welding paths that are Ushaped (100 mm down, 600mm right, 100mm up), as shown in Fig. 10.

The tests have shown that the quality of the weld by the SWERS is better than the manual FCAW (fluxed-core automated welding) and SMAW (stick welding) in terms of uniformity, shape and consistency. Macro-etching inspection and micro hardness tests have shown that the samples are free of porosity and cracks. For the cycle time, the SWERS also performed better than the manual processes. Results of a typical timing run are shown in Table 2. 


\begin{tabular}{|l|ccc|}
\hline \multicolumn{1}{|c|}{ Time } & SWERS & FCAW & SMAW \\
\hline Set-up & N/A & $60 \mathrm{sec}$ & $30 \mathrm{sec}$ \\
Teaching & $150 \mathrm{sec}$ & N/A & N/A \\
Welding & $234 \mathrm{sec}$ & $306 \mathrm{sec}$ & $608 \mathrm{sec}$ \\
\hline Total & $384 \mathrm{sec}$ & $366 \mathrm{sec}$ & $638 \mathrm{sec}$ \\
\hline
\end{tabular}

Table 2: Typical Cycle Times

The cycle time is expected to be even faster in actual workpieces where it has longer welding seams and repeated patterns. For such job, the PTP mode and "Teach-Weld-Weld" mode can be used so that the teaching time can be significantly reduced.

The flexibility of SWERS has also been demonstrated in pipe welding operations. Although not originally designed for this application, the general applicability of the walk-through teaching methods has made the application to pipe welding very straightforward with minimal effort.

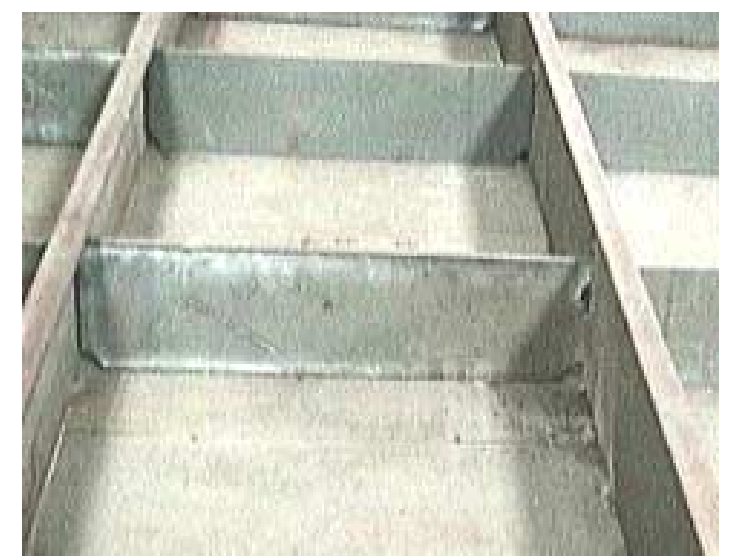

Fig. 10 Workpiece for Welding Test 


\section{CONCLUSIONS}

The biggest advantage of SWERS is the easier and faster operation compared to a conventional robotic system. With the implementation of the new Walk-Through Teach method, robot can now be used for the panel line for a faster welding time as shown in the welding tests. For a specific welding length of $1 \mathrm{~m}$, the total operation time including robot teaching time of SWERS is $5 \%$ faster than manual welding. It will be even faster if the workpiece have repeated patterns because the “Teach-Weld-Weld" mode can be used

Since the SWERS is so easy to operate, the training time required to use the system is much shorter than for a conventional robotic system. An operator with certain knowledge of welding process can be trained to use the system within one day.

Apart from the improvement of welding cycle time, the welding quality of the robotics system is better than manual arc welding due to the implementation of optimised welding parameters on the system and non-stop welding lines. Besides, the precise motion of the robot in addition to the arc sensing for search and tracking the seams have also contributed for a better welds.

As the actual welding is handled by the robot, the operator can now stay away from the fumes and heat generated by the welding. A less hazardous and better working condition for human is another advantage of using the robotic system.

By incorporating a force-torque sensor together with powerful algorithms, a new way of robot teaching method is implemented and proved useful for the automated welding in shipyards. The custom design man-machine interface is crucial for the operation of the robotic system for the complicated welding operation.

\section{ACKNOWLEDGEMENTS}

The support of the National Science Technology Board (Grant NSTB/17/3/16), and Keppel Far East Levingston Shipbuilding Ltd is gratefully acknowledged. This project is a collaboration between National University of Singapore, Gintic Institute of Manufacturing Technology and the Singapore Productivity and Standards Board. The contribution of the following other members of the project team are gratefully acknowledged: Ng Teck Chew, Kang Yanjun, Yang Jiandong, 
Fung Mok Wing, Danny Tan, Wee Teck Guan, Lee Weng Kee, Patrick Yung, Zeng Xiao Ming, Lim Tow Koon and Roy Lim.

\section{REFERENCES}

ABB (1993), "Arc welding is still very much a craft," ABB Robotics Review, No. 1.

Ang M. H. Jr and Andeen G. B. (1995), "Specifying and Achieving Passive Compliance Based on Manipulator Structure", IEEE Transactions on Robotics and Automation, Vol. 11, No. 4, pp. 504-515.

Buchal R O, et.al. (1989), Simulated off-line programming of welding robots, International Journal of Robotics Research, Vol 8, No 3, pp. 31-43.

Groover M P, Weiss M, Nagel R N, and Odrey N G (1986), Industrial Robotics Technology, Programming, and Applications, McGraw Hill, USA.

Hogan N. (1985), "Impedance Control: An Approach to Manipulation: Part I - Theory, Part II - Implementation, Part III Application", ASME Journal of Dynamic Systems, Measurement, and Control, Volume 107, pp. 1 - 24.

Kangsanant T, and Wang R G (1995), CAD-based robotic welding system with enhanced intelligence, in Proceedings of the Third International Conference on Computer Integrated Manufacturing, World Scientific Publishing, Singapore.

Khatib O. (1987), "A Unified Approach to Motion and Force Control of Robot Manipulators: The Operational Space Formulation". IEEE Journal of Robotics and Automation, Vol. 3, No. 1, pp 43-53.

Lawrence D. A (1988), "Impedance Control Stability Properties in Common Implementations," Proceedings of the 1988 IEEE International Conference on Robotics and Automation, pp. 1185 - 1190.

Paul R. P. (1981), Robot Manipulators - Mathematics, Programming and Control, MIT Press, USA.

Pelletier M. and Doyon M (1994), "On the Implementation and Performance of Impedance Control on Position Controlled Robots", Proceedings of the 1994 IEEE International Conference on Robotics and Automation, pp. 1228 - 1233.

PSB (1990), Singapore Standard CP53: 1990: The Safe Use of Industrial Robot. Singapore Productivity and Standards Board.

Roedsted, J. and Koch, B. (1987), “BRITE 1543: Flexible Low Cost Automation of Arc Welding," $2^{\text {nd }}$ Int. Conf. on Developments in Automated and Robotic Welding, England, pp. 55-68.

Salisbury J K (1980)." Active Stiffness Control of a Manipulator in Cartesian Coordinates, ". 19 ${ }^{\text {th }}$ IEEE Conference on Decision and Control. 
Skjolstrup C E and Ostergaard S (1994), Shipbuilding using automated welding processes, Welding International Review, February, pp. 213-217.

Tan T K, Ang M H Jr. and Teo C L (1997), "Non-Model-Based Impedance Control of an Industrial Robot", in Proceedings of the International Conference on Advanced Robotics, USA, pp 407-412.

Weston, J., Editor (1989), Exploiting Robots in Arc Welded Fabrication, The Welding Institute. 